\title{
CMOS PHASE SHIFTER FOR CONFORMAL PHASED ARRAY BEAMFORMER APPLICATIONS
}

\author{
A Thesis \\ Submitted to the Graduate Faculty \\ of the \\ North Dakota State University \\ Of Agriculture and Applied Science
}

\author{
By \\ Alarka Sanyal \\ In Partial Fulfillment of the Requirements \\ for the Degree of \\ MASTER OF SCIENCE \\ Major Department: \\ Electrical and Computer Engineering
}

April 2015

Fargo, North Dakota 


\section{North Dakota State University \\ Graduate School}

Title

CMOS PHASE SHIFTER FOR CONFORMAL PHASED ARRAY

BEAMFORMER APPLICATIONS

\section{By}

Alarka Sanyal

The Supervisory Committee certifies that this disquisition complies with

North Dakota State University's regulations and meets the accepted

standards for the degree of

\section{MASTER OF SCIENCE}

SUPERVISORY COMMITTEE:

Dr Debasis Dawn

Chair

Dr Scott C. Smith

Dr Gursimran S. Walia

Approved:

$\frac{5 / 14 / 2015}{\text { Date }} \quad \frac{\text { Dr Scott C. Smith }}{\text { Department Chair }}$




\begin{abstract}
A vector modulator based phase shifter is developed using 0.18um CMOS process at Sband frequency to be integrated into a conformal phased array antenna to recover the desired radiation pattern in the entire $360^{\circ}$ range. The phase shifter has a variable gain amplifier integrated into the circuit in order to vary gain along with phase for precise control to correct the degraded radiation pattern due to the conformal shaping. The results show state-of-the-art performances including more than $7 \mathrm{~dB}$ conversion gain with variable feature, a continuous phase rotation of $360^{\circ}$ with steps as low as $11.25^{\circ}$ and very low power consumption of $17 \mathrm{~mW}$, for the first time to the best of the authors' knowledge. The chip size including all pads is $1.5 \mathrm{~mm} \times 0.75 \mathrm{~mm}$.
\end{abstract}




\section{ACKNOWLEDGEMENTS}

Firstly, I would like to thank my academic advisor, Dr. Debasis Dawn for his continuous support, patience and guidance in helping me complete my research work.

I would also like to thank Alfonso Mendoza Radal for his support and guidance while working on my research work.

ND NASA EPSCoR has supported this project under the agreement FAR0020852. I would like to thank Prof. Benjamin D. Braaten of North Dakota State University, Fargo, ND, Dr. Neil F. Chamberlain of NASA JPL, Dr. Michael T. Reich of Center for Nanoscale Science and Engineering (CNSE) of North Dakota State University, Fargo, ND and Prof. Dimitris E. Anagnostou of Department of Electrical and Computer Engineering, South Dakota School of Mines and Technology, Rapid City, SD for their support and collaboration.

I am very grateful to all my committee members, Dr Scott C. Smith, Dr Gursimran S. Walia and my advisor Dr Debasis Dawn for their patience and support.

I would also like to thank all my colleagues at NDSU for their kind help and support during the course of my Master's program. I am really grateful to all these people and deeply appreciate the support offered by them. 


\section{DEDICATION}

I would like to dedicate this thesis to $M a$ and $B a b a$ for their unconditional love and support. 


\section{TABLE OF CONTENTS}

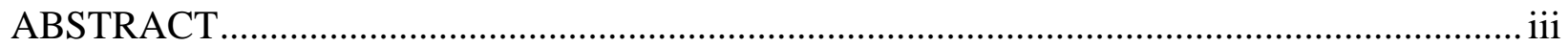

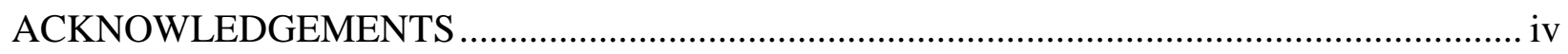

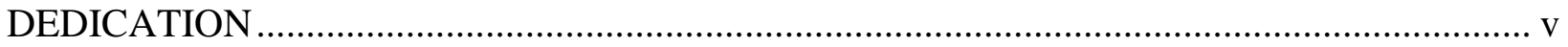

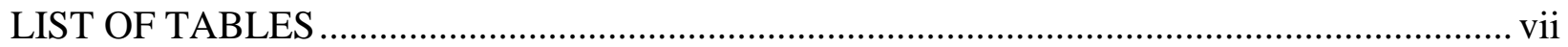

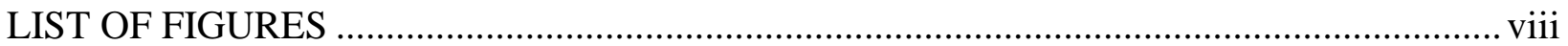

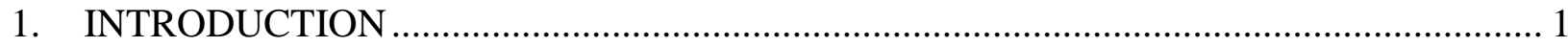

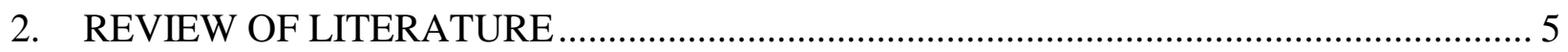

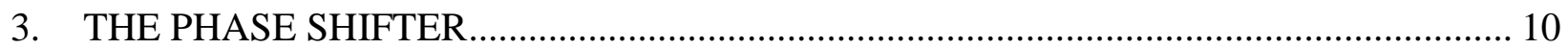

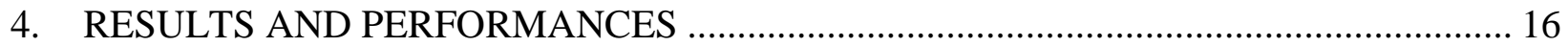

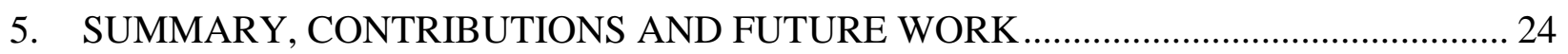

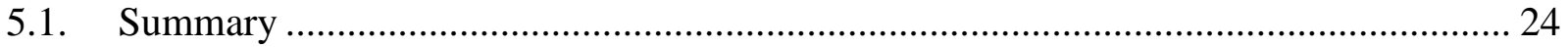

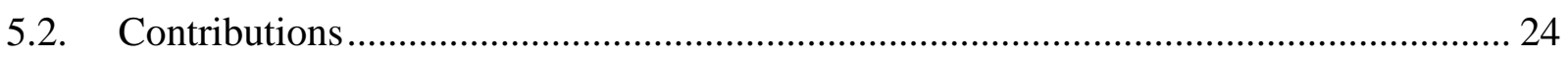

5.3. Suggestions for Future Work ………………………............................................ 25

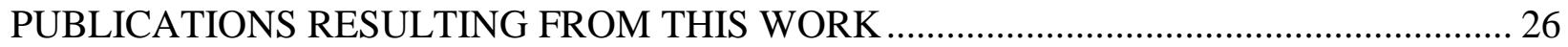

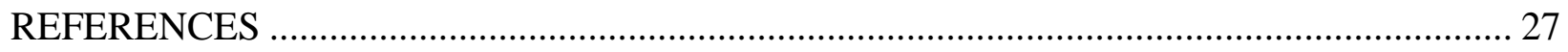




\section{LIST OF TABLES}

$\underline{\text { Table }} \quad \underline{\text { Page }}$

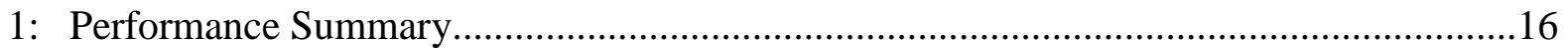

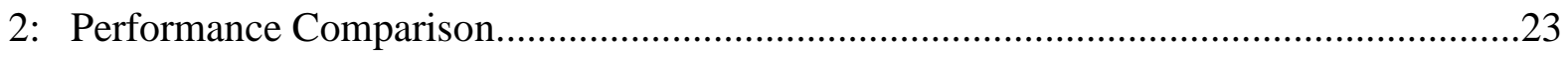




\section{LIST OF FIGURES}

$\underline{\text { Figure }}$

$\underline{\text { Page }}$

1: A single antenna element and an antenna array ....................................................

2: Integrated Beam Former with antenna array and phase shifters ..................................2

3: Integrated CMOS phase shifter with precise gain/phase control ..................................

4: The circuit schematic of the Gilbert cell phase shifter integrated with the variable gain

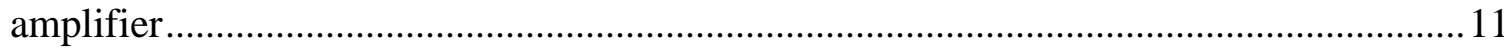

5: Phase control mechanism.................................................................................. 12

6: The circuit schematic of the passive hybrid.......................................................... 13

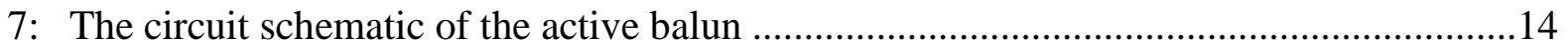

8: The gain variation of the circuit for single ended output with a particular phase setting...17

9: Phase shifting performance over $0^{\circ}$ to $180^{\circ}$ with $22.5^{\circ}$ steps ......................................18

10: Frequency characteristics of phase variation in the entire $360^{\circ}$ range ...........................19

11: S-parameter analyses of conversion gain and return loss ........................................20

12: Stability of conversion gain and return loss over phase variation ...............................21

13: Large-signal performance showing compression of the integrated phase shifter.............22

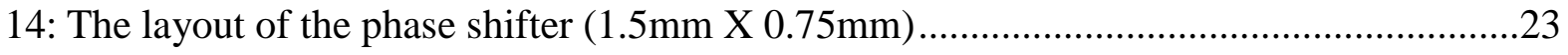




\section{INTRODUCTION}

Phase shifters are critical components of any phased array antenna system [1] which are of particular interest for NASA in wireless communications involving space suits worn by astronauts and is found to be very popular because it allow the scanning of the antenna beam electronically. The phase shifters can be used to change the angle of radiation of transmitted and received waveform signals, thus avoiding bulky arrangements for mechanical rotation of the antenna system. To get a broadside radiation pattern in a microstrip antenna array, all the antenna elements must be fed with same voltage phase.

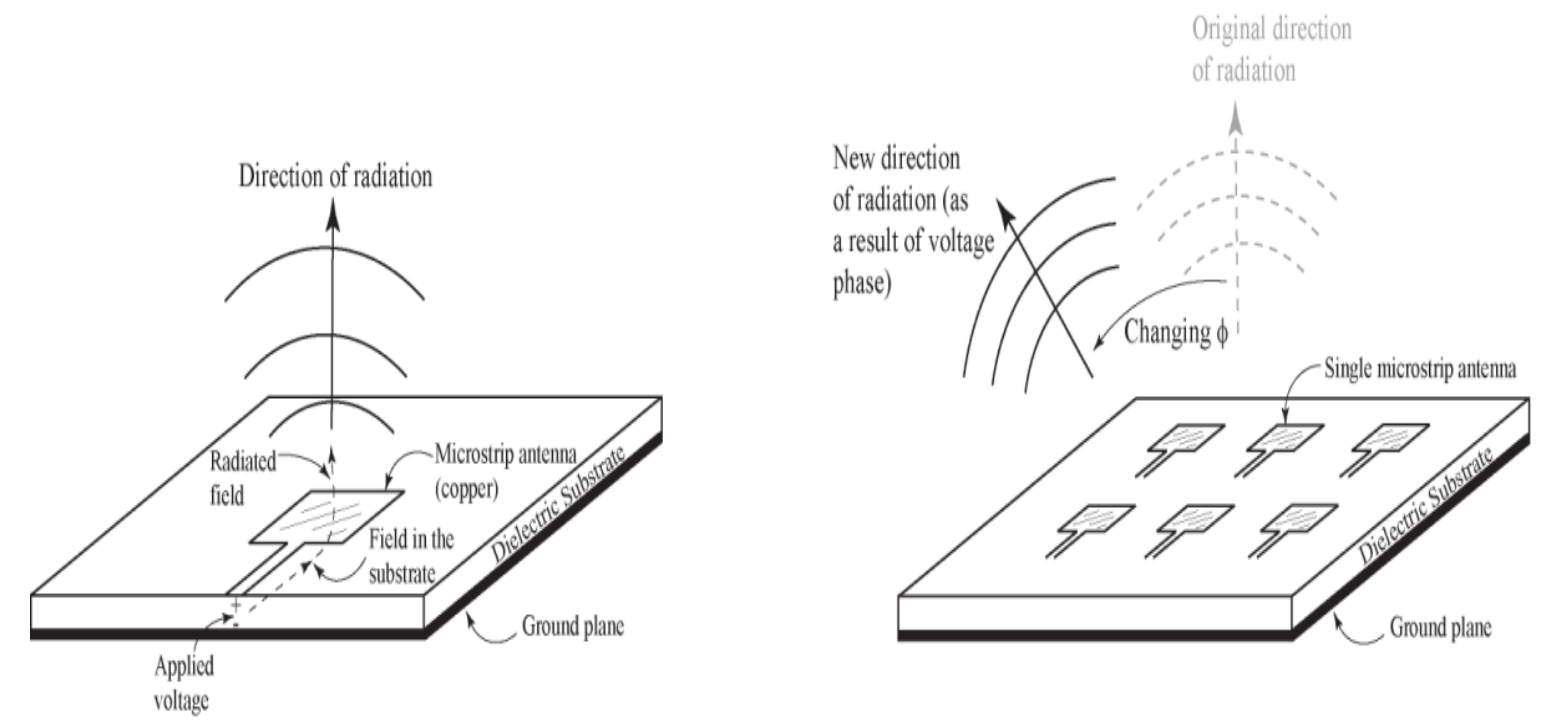

Figure 1: A single antenna element and an antenna array 
However, when this antenna array is bent or placed on a conformal surface, the radiation pattern is changed from its broadside direction, and overall performances such as angle of radiation and gain are degraded. The amount of degradation depends upon the bending radius. In order to restore the original radiation pattern, appropriate phase compensation is required on each antenna array element [2]. The CMOS phase shifter described here can be integrated with a conformal phased array antenna to fully recover the radiation pattern through precise gain and phase control mechanism.

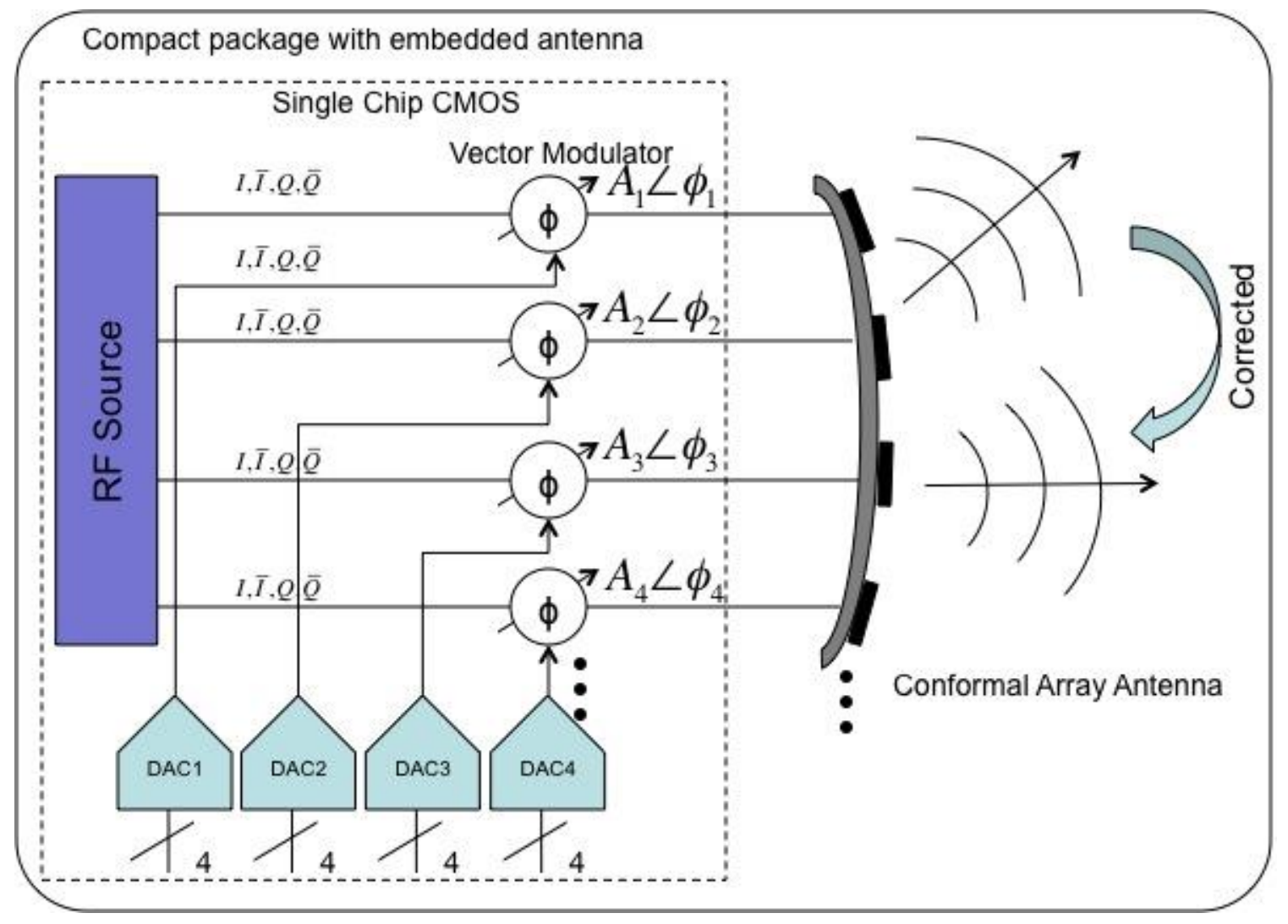

Figure 2: Integrated Beam Former with antenna array and phase shifters 
Phase shifters can be classified into passive and active phase shifters [3], with the former having the disadvantage of signal attenuation, which can be overcome by using the active phase shifters [4]. Other way to classify the phase shifters is in analog and digital [5]; analog phase shifters provide continuous phase variation over a certain range and are controlled by a varying voltage. Digital phase shifters provide fixed phase steps that are selected using a binary coded input [5].

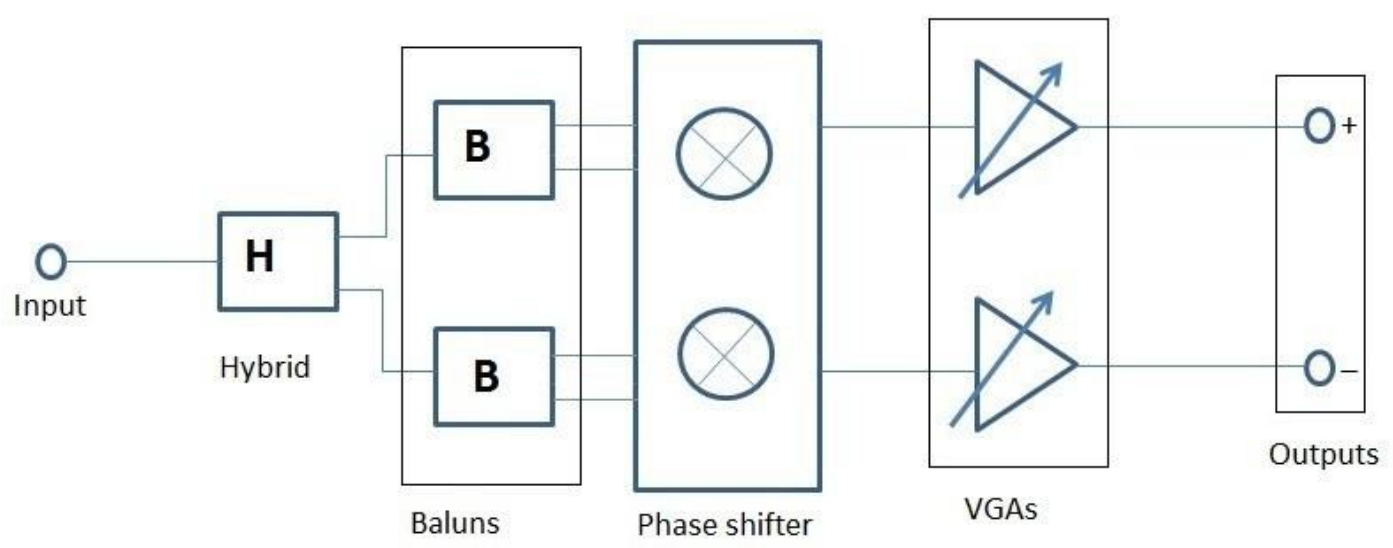

Figure 3: Integrated CMOS phase shifter with precise gain/phase control

For example, a $360^{\circ}$ digital phase shifter with 4 bits control can obtain discrete phase-shift steps of $22.5^{\circ}$, whereas an analog phase shifter can produce a continuous phase-shift over the $360^{\circ}$ range. Phase shifters can be designed in different forms, namely switched line phase shifters [7], loaded-line phase shifters [8], and reflection-type phase shifters [9].

Due to the rapid scaling of the transistor lengths in the recent past, it has become possible to fully integrate large number of transistors with both analog and digital functionality into a single chip [6]. Especially CMOS RFIC (Radio Frequency Integrated Circuits) made possible towards 
realization of system-on-chip (SOC) solutions where all analog and digital functionality can be integrated into a single process platform and analog radio can be controlled through digital circuitry with programs written on a computer. Smaller chip area also helps in energy consumption, as well as the cost reduction. The reduced power requirement opens the possibility for wide applications including portable electronics.

Here, we have proposed an integrated CMOS phase shifter, which can be operated at Sband frequency. The phase shifter consists of passive hybrid, active balun and a variable gain amplifier with buffer stages integrated into a single chip which can be integrated into a conformal phased array beamformer to achieve precise gain/phase control simultaneously. The phase shifter is designed in 0.18um CMOS process. 


\section{REVIEW OF LITERATURE}

Phase shifters form a very important part of phased array systems [1]. Microwave phase shifters were previously implemented in III-V compound semiconductors for stringent performance requirements. With the current developments in fabrication technology, more and more monolithic microwave integrated circuits (MMICs) are now fabricated in CMOS technologies [16], [17], although the substrate losses and inferior Q-factors of the passive components still hinder the realization of CMOS phase shifters at microwave frequencies. To consider the hardware cost and system integration, it is desirable to implement high-performance microwave phase shifters using a standard CMOS process [15]. A change in the direction of transmission of an antenna array can be caused due to a various reasons. In order to compensate the change in direction or in to get the transmission of the radiating beam in a desired direction, a phase shift can be applied to the input signal of the antenna. This is where phase shifters come into

play. The goal of designing a good phase shifter is to have a low insertion loss and also a gain in the output amplitude.

There are different architectures for implementing phase shifters such as reflection-type phase shifters [14], switched-line phase shifters [7], loaded-line phase shifters [8] and vector sum based phase shifters [11]. The phase shifters are chosen based on performance specifications like phase and amplitude imbalance, insertion loss, area, power, etc.

Shunt and series transmission lines together with reactive elements like varactors or diodes are used to design Passive phase shifters. The major advantages of this type of phase shifters are the simplicity of implementation and zero DC power consumption [3]. The major disadvantages 
are higher insertion loss and obtaining more than $180^{\circ}$ phase shift at mm-wave frequency using the low quality passive phase shifters of standard digital CMOS processes. One of these disadvantages of obtaining a phase shift over $180^{\circ}$ is achieved by designing Active phase shifters which can obtain phase shifts over the entire $360^{\circ}$ range. Active phase shifters consist of a quadrature generator along with a weighed I/Q summer called Vector Modulator (VM) and since the output is obtained by the addition of the quadrature signals, any arbitrary phase shift over the entire range of $360^{\circ}$ can be obtained [3].

[18] implemented a distributed phase shifter with diode-loaded transmission line, which is widely used due to its low power consumption and low insertion loss, although the biggest drawback for this architecture is the considerably large chip area, especially for applications below $10 \mathrm{GHz}$ frequency. To overcome this drawback of large circuit size, [13] implemented an ultracompact phase shifter by using spiral inductors to replace the required transmission line sections, although it had its own set of drawbacks as it suffered from higher insertion loss and limited phase shift range.

The reflection-type phase shifter (RTPS), utilizing a 3-dB hybrid coupler and a pair of reflective termination circuits with impedance transformers is also widely used for its wide-band performance [14], [19]-[21], although the bulk silicon processes, the low inductor/varactor $Q$ values and the limited varactor capacitance range produce high losses and small phase control ranges [22]. Another way of obtaining a broad-band phase shifter is by switching between a lowpass filter and a high-pass filter, which is called a switching-type phase shifter (STPSs). The low resistivity substrate and large on-state resistance in CMOS process will degrade the performance of the switching-type phase shifters [23]. The two types of phase shifters, RTPS and STPS can be cascaded for multibit operation, which increases their insertion losses and phase errors [19]. 
Compared to this, active phase shifters using vector sum method can provide continuous phase shift or multibit operation with low loss or gain [24]-[30].

[31] implements two K-band monolithic phase shifters, the Cartesian phase shifter and the hybrid polar phase shifter. The Cartesian phase shifter can achieve a $360^{\circ}$ phase shift by using the vector sum of four orthogonal signals of which amplitudes can be varied over a wide dynamic range. It has a chip size of $0.95 \mathrm{~mm} X 0.76 \mathrm{~mm}$, which was achieved by reducing the number of couplers and delay lines. It has a continuous phase shift and an insertion loss of $8 \mathrm{~dB}$ with a $37-\mathrm{dB}$ dynamic range between 15-20 Ghz. The hybrid polar phase shifter on the other hand separates the input signal into two paths of which one can be placed anywhere in the first quadrant and the other one can cover the whole third quadrant, combining which, again a continuous phase shift can be achieved. It has an insertion loss of $16.2 \mathrm{~dB}$ with a $38.8-\mathrm{dB}$ dynamic range from $15-20 \mathrm{GHz}$. The main drawbacks of these phase shifters are they have losses instead of gain in amplitude, limited output power and degraded noise figure due to the insertion loss and nonlinearity.

SiGe BiCMOS technology at 65 -nm node and beyond is also ideally suited for highly integrated and low-power W-band phased arrays which helps in new system architectures based on electronic beam-forming and beam-steering [32]. These help in increased range, resolution and zooming functions for applications such as automotive and industrial sensors, active and passive imaging. [32] describes $70-77 \mathrm{GHz}$ and $80-94 \mathrm{GHz}$ phase shifters with 3-dB bandwidths. The phase shifters use the phase interpolation architecture consisting of a $90^{\circ}$ hybrid which separates out the input signal into two equal-amplitude paths in quadrature and a phase rotator. The $77 \mathrm{GHz}$ phase shifter uses BiCMOS technology whereas the $94 \mathrm{GHz}$ phase shifter uses CMOS technology. Both the designs have VGAs, which are obtained by telescopic-cascode Gilbert cells and current 
summer implemented as a differential-to-single-ended balun. The gain, isolation and bandwidth are maximized by applying the mm-wave signal at the gates of the common-source devices.

Even though the different distributed types of phase shifters like switched transmission lines [33]-[35], 90-hybrid coupled lines [36]-[38], and periodic loaded lines [39]-[41] can achieve true time delay along the line sections, their physical sizes make them impractical for integration with multiple arrays in a commercial IC process, especially below K-band frequencies. The physical dimensions of phase shifters are reduced by the migrations from distributed networks to lumped-element configurations, such as synthetic transmission lines with varactors tuning [42][44], lumped hybrid-couplers with reflection loads [12], [22], [45] and the combined topologies of lumped low-pass filters and high-pass filters [46]-[48]. However, for fine phase quantization levels over wide operation bandwidth, the size of the lumped passive networks increases a lot, the main reason for which is the use of the various on-chip inductors, and is unsuitable for integrated phased array systems on a chip. Also, in most cases, there is a nonlinear relationship between the control signal (voltage or current) and output phase of the lumped passive phase shifters, which makes the design of the control circuits quite complex [49]. The passive phase shifters do not consume any DC power and still can achieve good linearity, but their large insertion loss requires an amplifier to compensate the loss, typically more than two stages at high frequencies $(10 \mathrm{GHz})$, which offsets the major merits of good linearity and low power dissipation of the passive phase shifters. In comparison to the passive phase shifters, active phase shifters [50]-[51], [29], [31], [52]-[55] can achieve a high integration level with decent gain and accuracy along with a fine digital phase control with a required power budget where differential phases are obtained using transistors instead of passive networks. Different phase shifters like endless PS [50], a programmable PS [21], a Cartesian PS [31], or a phase rotator [52], the underlying principle for all cases is to interpolate 
the phases of two orthogonal-phased input signals by adding the I/Q inputs for synthesizing the required phase. The different amplitudes between the I- and Q-inputs result in different phases. Thus, the basic function blocks of a typical active phase shifter are composed of an I/Q generation network, an analog adder, and control circuits which set the different amplitudes of I- and Q-inputs in the analog adder for the necessary phase bits [11].

A CMOS 4-bit active digital phase shifter is presented in [11] for $\mathrm{X}-$, $\mathrm{Ku}-$, and $\mathrm{K}$-band frequencies. The phase shifters interpolate the phases of the quadrature input signals by adding two I/Q inputs. Excellent signal, precision, increased operating bandwidth and minimal loss are obtained by developing resonance-based differential quadrature networks. 


\section{THE PHASE SHIFTER}

A double balanced Gilbert cell has been used for designing this phase shifter as shown in Figure 3 (block diagram) and in Figure 4 (circuit schematic). In the bottom part, we have the vector modulator circuit with the Variable Gain Amplifier (VGA) circuit in the center and the two buffer circuits on each side. Each small block of the vector modulator has two top transistors for passing the RF signal and a tail transistor at the bottom to pass the control voltages. The common gate and common source transistors connected in the cascade topology form each small block of the vector modulator. The control voltages applied at the bottom tail transistors of the vector modulator help in passing the RF signal, being fed to the top transistors, to the output of the vector modulator circuit, which is then passed on to the input of the VGA circuit. The VGA circuit also has a pair of top transistors, which are used as the input for the phase changed signals from the vector modulator circuit, and a tail transistor at the bottom to control the amplitude of the signal. By controlling and varying the control signal of the bottom transistor, we can vary the output amplitude of the RF signal. The output signal is then passed through the buffer circuits. The buffer circuits are meant for $50 \Omega$ output matching during measurements. 


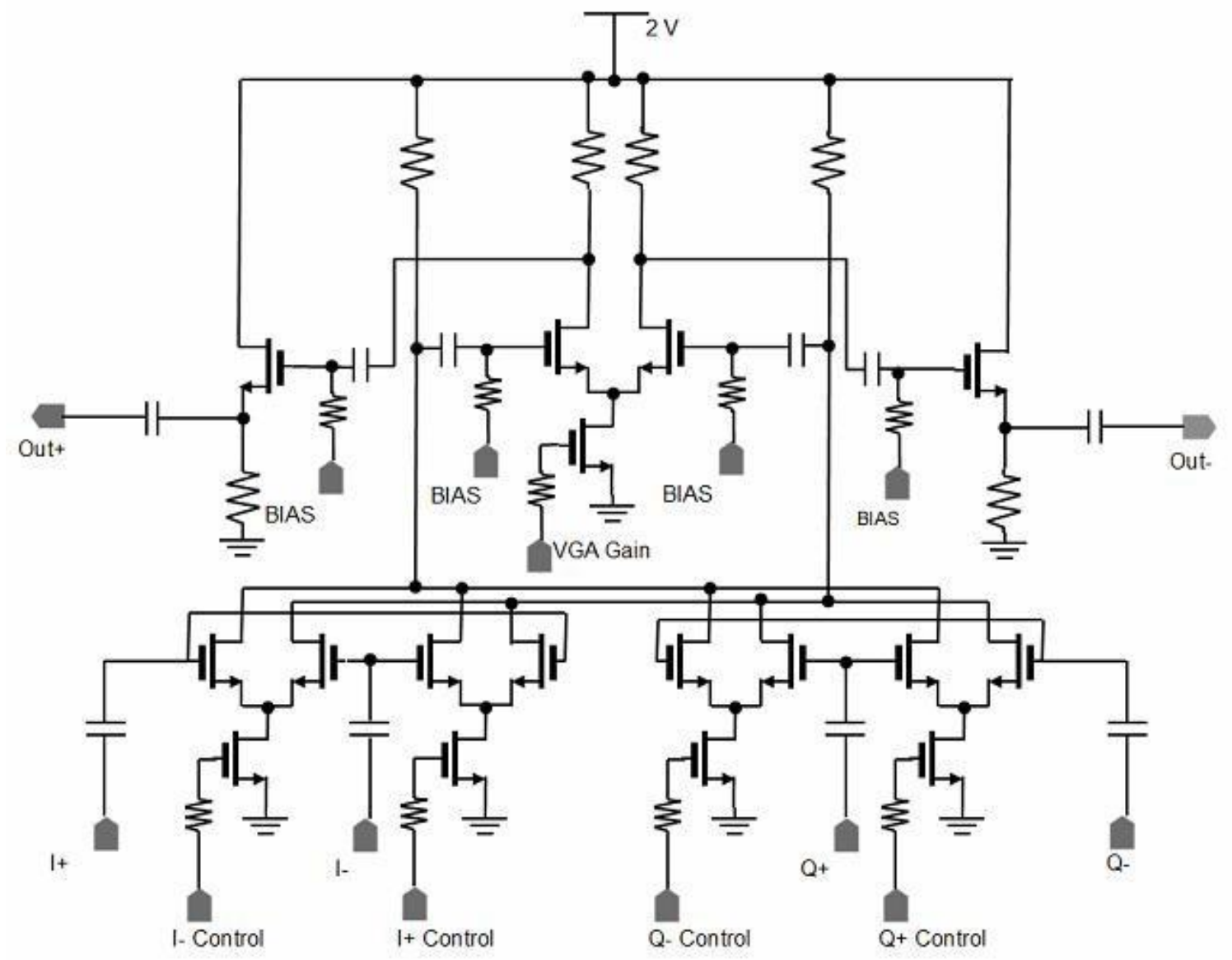

Figure 4: The circuit schematic of the Gilbert cell phase shifter integrated with the variable gain amplifier

This integrated phase shifter design is optimized through co-design method where each connecting block's input/output impedances are considered in the design, thus, maximizing the performance of the overall chip while minimizing power consumption. Figure 5 shows the principle of operation for the vector modulator based phase shifter. 


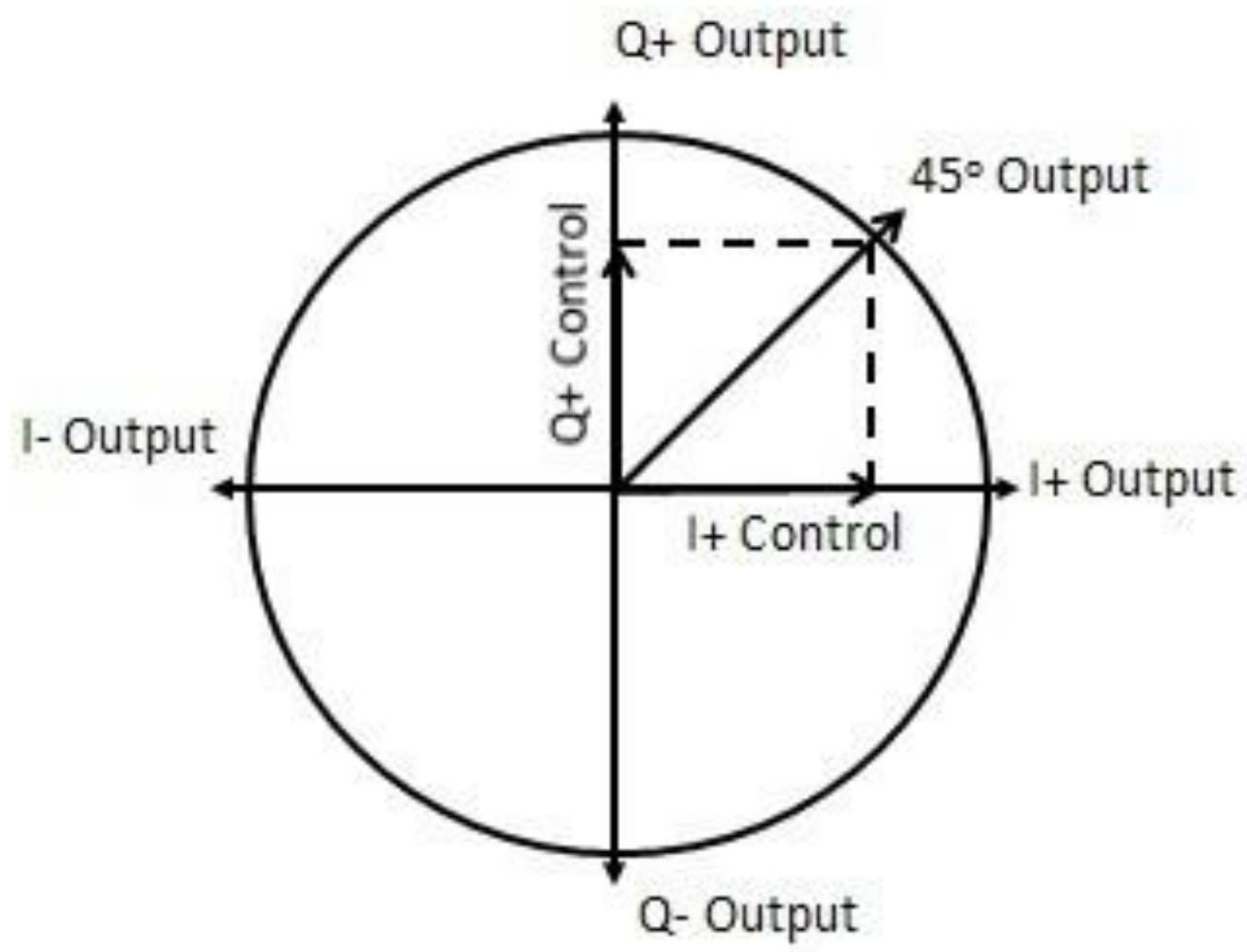

Figure 5: Phase control mechanism

Starting with 4 signals with phases of $0^{\circ}(\mathrm{I}+), 90^{\circ}(\mathrm{Q}+), 180^{\circ}(\mathrm{I}-)$ and $270^{\circ}(\mathrm{Q}-)$, the output signal with a specific phase is achieved by adding two of these input signals. By controlling the amplitude of each added signal, a continuous phase shift is possible. An on-chip passive hybrid (Figure 6) and an active balun circuitry (Figure 7) were included in the design to generate the required phases from the input $\mathrm{RF}$ signal. 


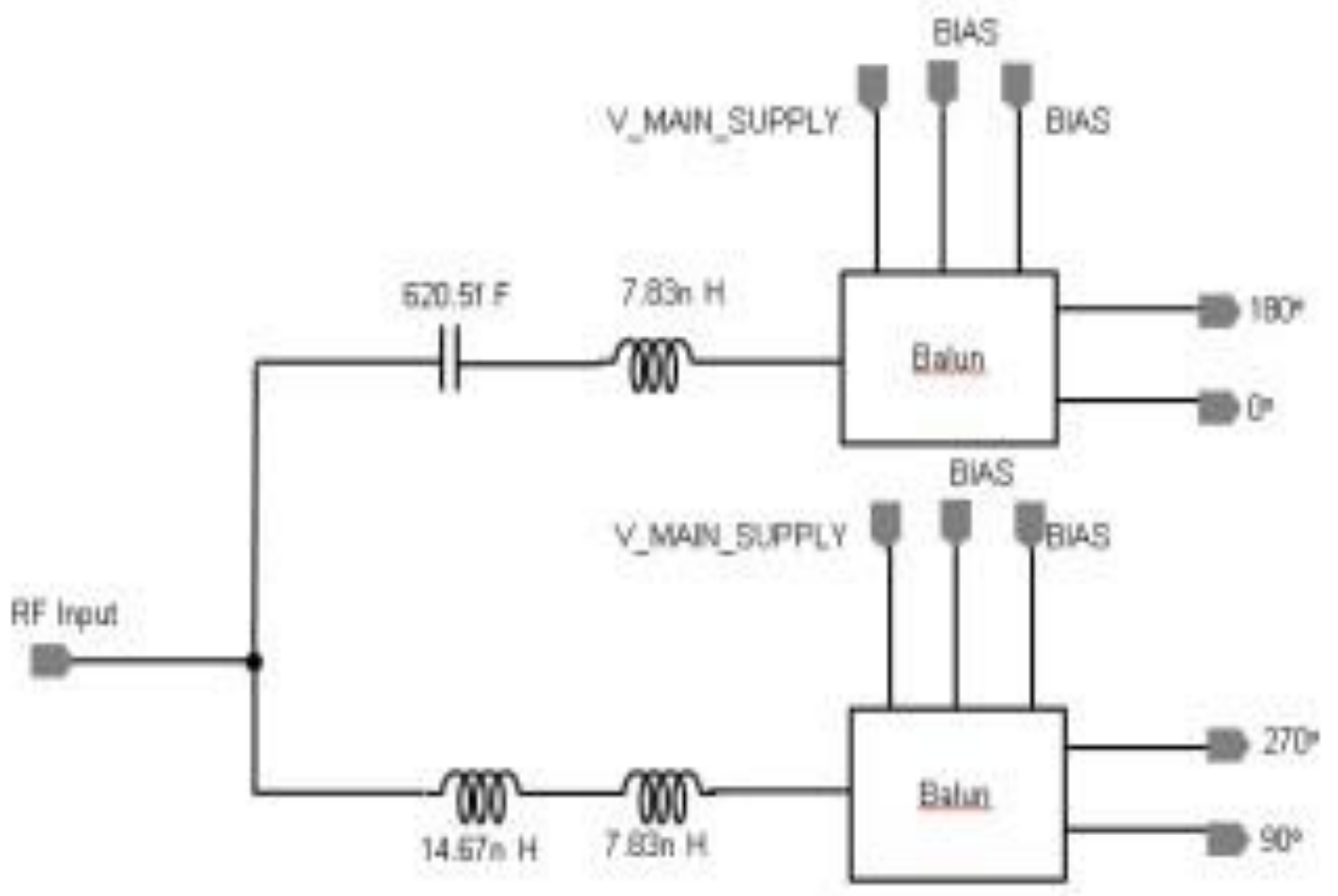

Figure 6: The circuit schematic of the passive hybrid 


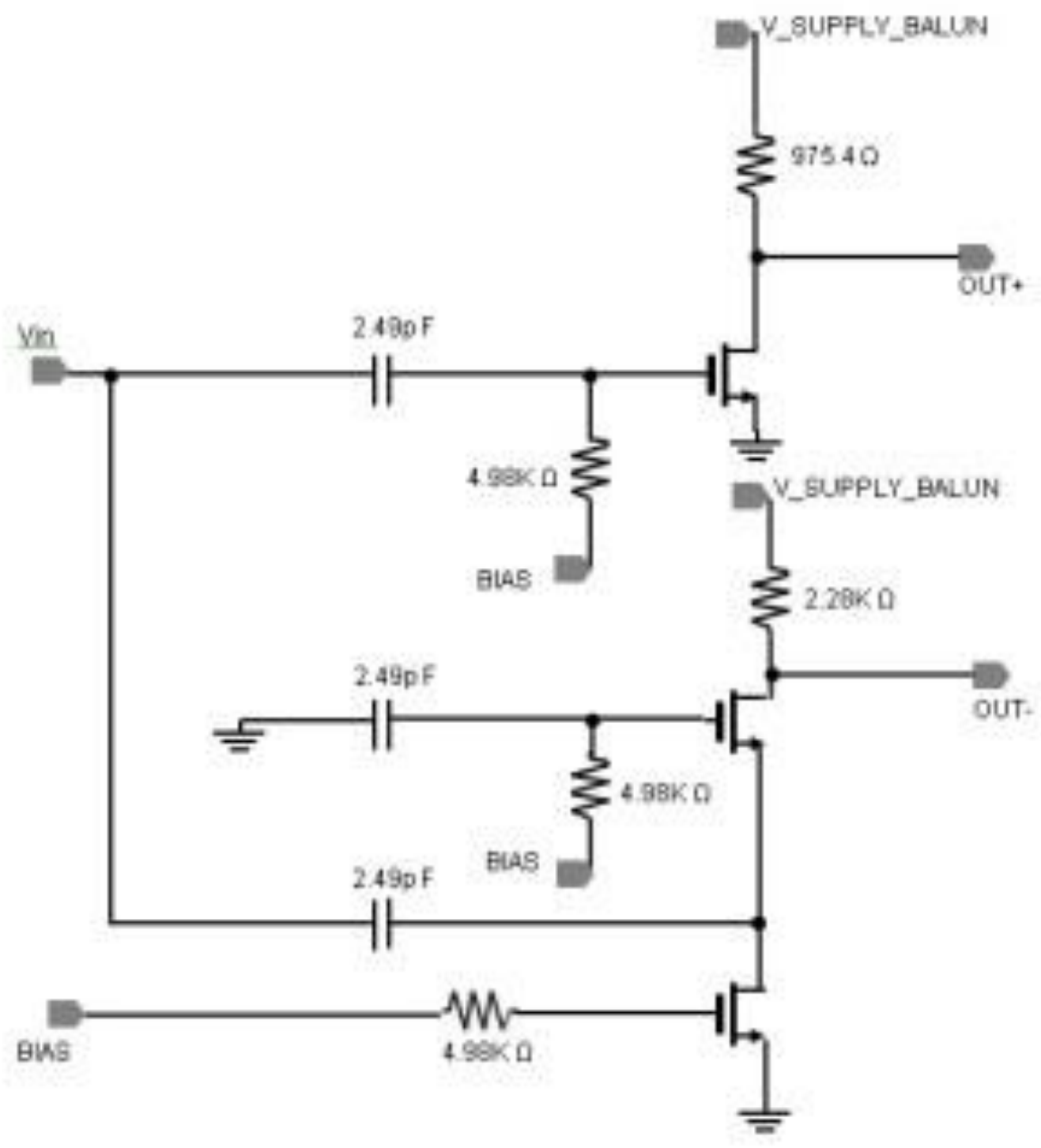

Figure 7: The circuit schematic of the active balun

The input RF signal is passed through a passive hybrid to produce I (in phase) and Q (quadrature) signals with a phase difference of $90^{\circ}$. The inductors and capacitor of the hybrid circuit helps in achieving the two signals with the phase difference as mentioned. The hybrid being designed with passive elements produces a loss in the amplitude of the RF signal when passed through it. This I \& $\mathrm{Q}$ signals are then fed to an active balun consisting of a common- 
source/common-gate topology and cascade topology, producing differential signals from each input signal, thus producing four separate RF signals with $90^{\circ}$ phase difference between each. The active balun compensates the losses incurred in the passive hybrid and produces extra gain. The amplitude of the signals is adjusted by using the bias levels of the active balun. All four RF signals from the balun (I+, I-, Q+, Q-) are then fed to the double-balanced Gilbert cell vector modulator. The input signals are fed to the upper differential pair transistors of the vector modulator. The four controlling voltages are fed to the tail transistors in order to control the phases of the output signal. The control of the output phase is accomplished by applying the proper voltages to any two of the four controlling ports so that signals can be passed through any two of the small blocks of the vector modulator and we can do the vector sum of the passed signals to achieve the required phase shift in the output vector added signal. For example, in order to get a phase shift of $45^{\circ}$, control voltage values must be given to the control ports I+ Control and Q+ Control, as shown in Figure 3. By varying all four-control voltage coefficients of these ports, any arbitrary phase shifts can be generated. The outputs of the Gilbert cell vector modulator are passed through a two stage VGA (Variable Gain Amplifier) in order to obtain an amplified output signal with variable gain capability. All the circuit blocks shown in Figure 3 are designed using the co-design methodology where input/output impedances are properly considered for RF matching ensuring the most efficient line-up performances with minimum chip size. 


\section{RESULTS AND PERFORMANCES}

Simulation is carried out using CADENCE® Spectre® [10] simulator and layout is done using Virtuoso ${ }^{\circledR}$ tool. The input to the hybrid and the output buffers are matched to $50 \Omega$. The performance summary of the phase shifter is shown in Table 1, which confirms state-of-the-art performances.

Table 1: Performance Summary

\begin{tabular}{|l|l|}
\hline Technology & $0.18 \mathrm{um} \mathrm{CMOS}$ \\
\hline Frequency & $2 \sim 3 \mathrm{GHz}$ \\
\hline Phase Range & $360^{\circ}$ \\
\hline Gain & $+7 \mathrm{~dB} @ 2.2 \mathrm{GHz}$ (variable) \\
\hline Input Return Loss & $<-25 \mathrm{~dB} @ 2.2 \mathrm{GHz}$ \\
\hline Output Return Loss & $<-11 \mathrm{~dB} @ 2.2 \mathrm{GHz}$ \\
\hline Power Consumption & $17.48 \mathrm{~mW}$ \\
\hline Chip Area & $1.5 \mathrm{~mm} \mathrm{X} 0.75 \mathrm{~mm}$ (with pads) \\
\hline
\end{tabular}




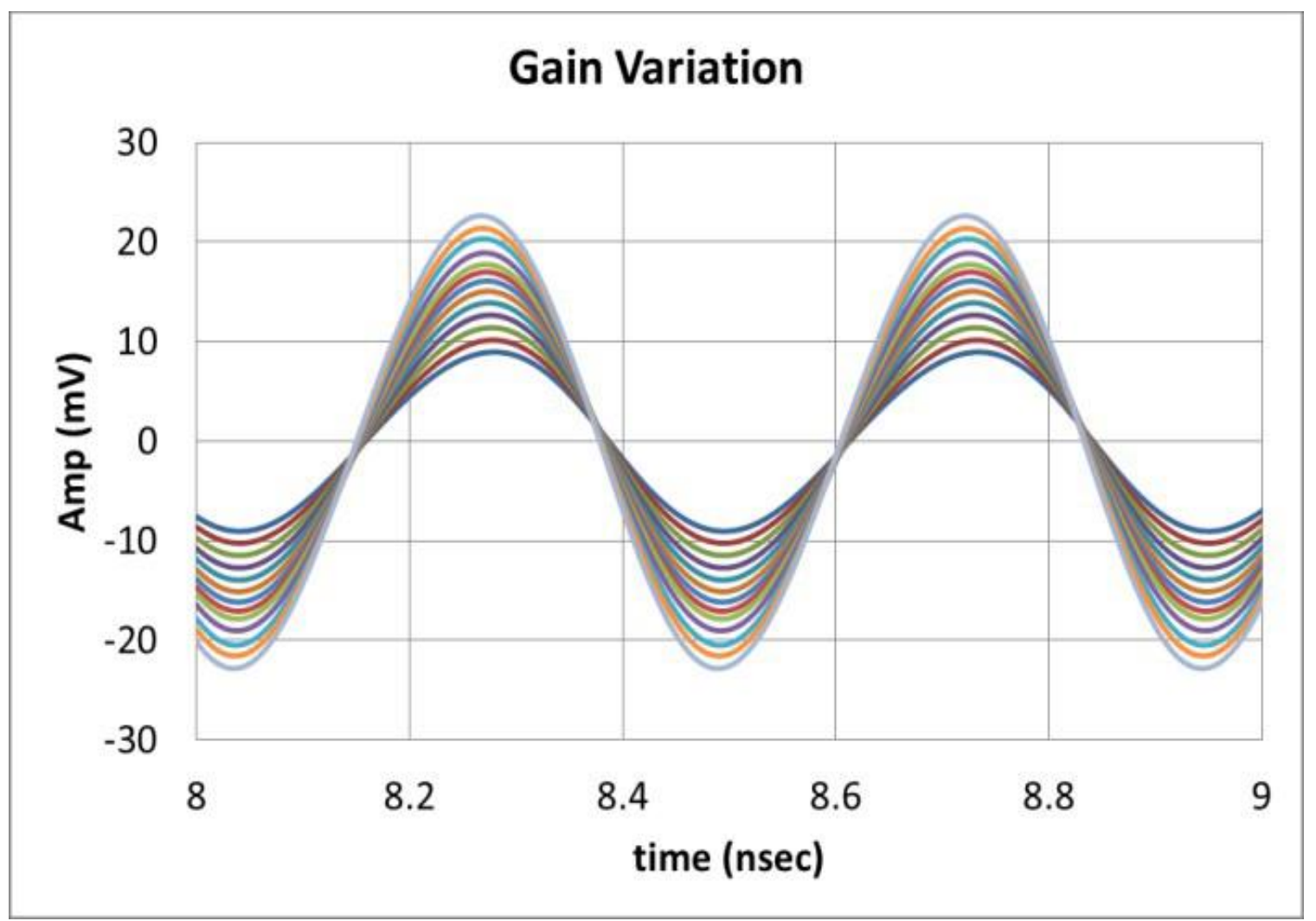

Figure 8: The gain variation of the circuit for single ended output with a particular phase setting

The circuit has been simulated with a $2.2 \mathrm{GHz}$ input RF signal at $-30 \mathrm{dBm}(10 \mathrm{mV}$ peak) amplitude which gives a maximum amplified output signal of $\sim 20 \mathrm{mV}$ peak for single ended output. We can also get the differential output from the two output ports from the two buffers of the circuit. From Figure 8, we can see that the circuit also produces $\sim 40 \mathrm{mV}$ peak for differential output. The gain variation for single ended output has been shown in Figure 8, which also confirms the stability at the desired phase setting. 


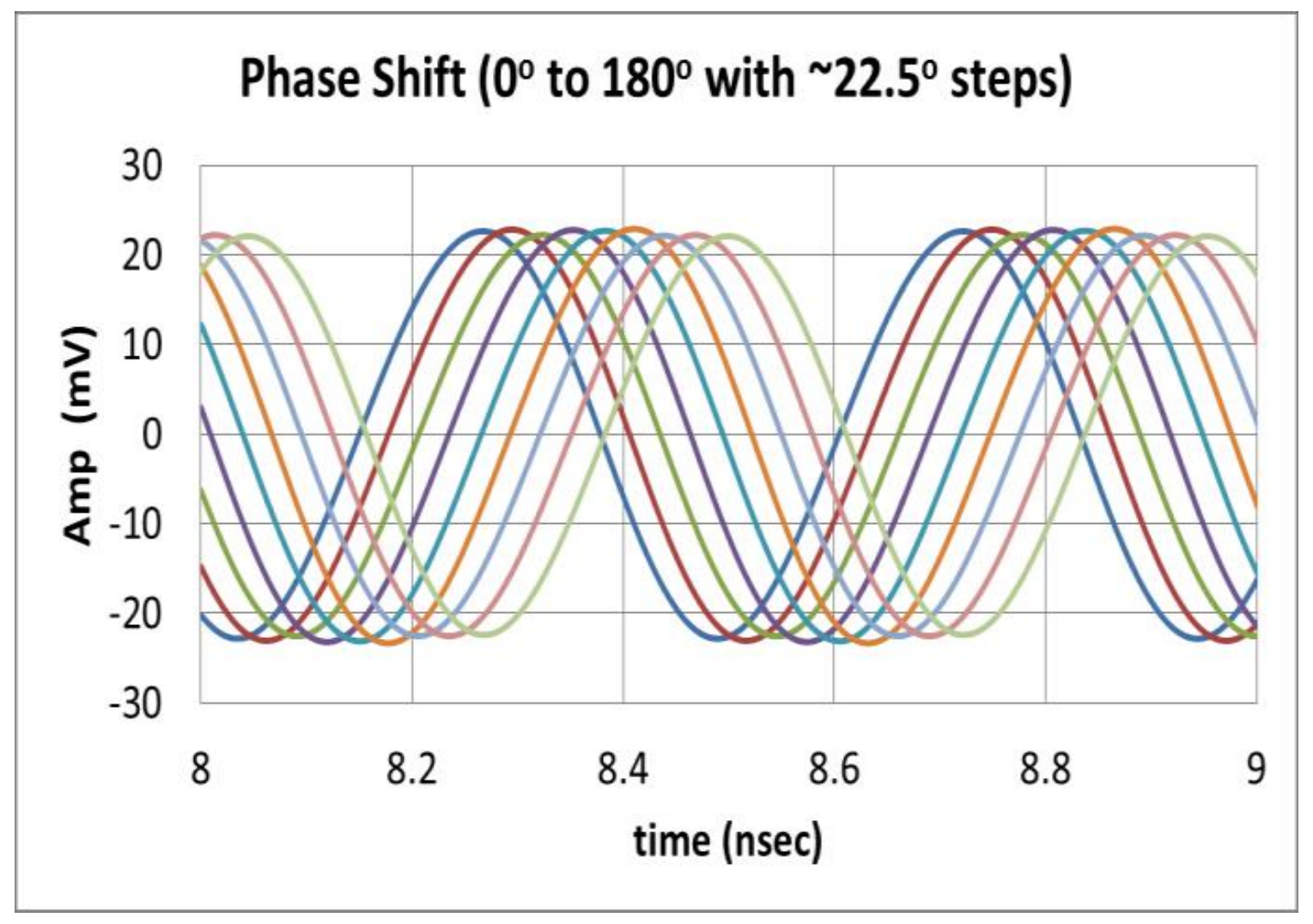

Figure 9: Phase shifting performance over $0^{\circ}$ to $180^{\circ}$ with $22.5^{\circ}$ steps

Figure 9 shows the phase shift performance of the circuit from $0^{\circ}$ to $180^{\circ}$ with $22.5^{\circ}$ steps while maintaining same amplitude. The phase shifter has the capability of continuously changing the phase in the $0^{\circ}$ to $360^{\circ}$ range, as shown in the frequency domain plot in Figure 10 . Though this phase shifter can achieve any arbitrary value of phase shift, the $22.5^{\circ}$ step is chosen to correlate the analog phase shifter performance through a digital bit control circuitry in terms of $2 \mathrm{n}$ relationship. 


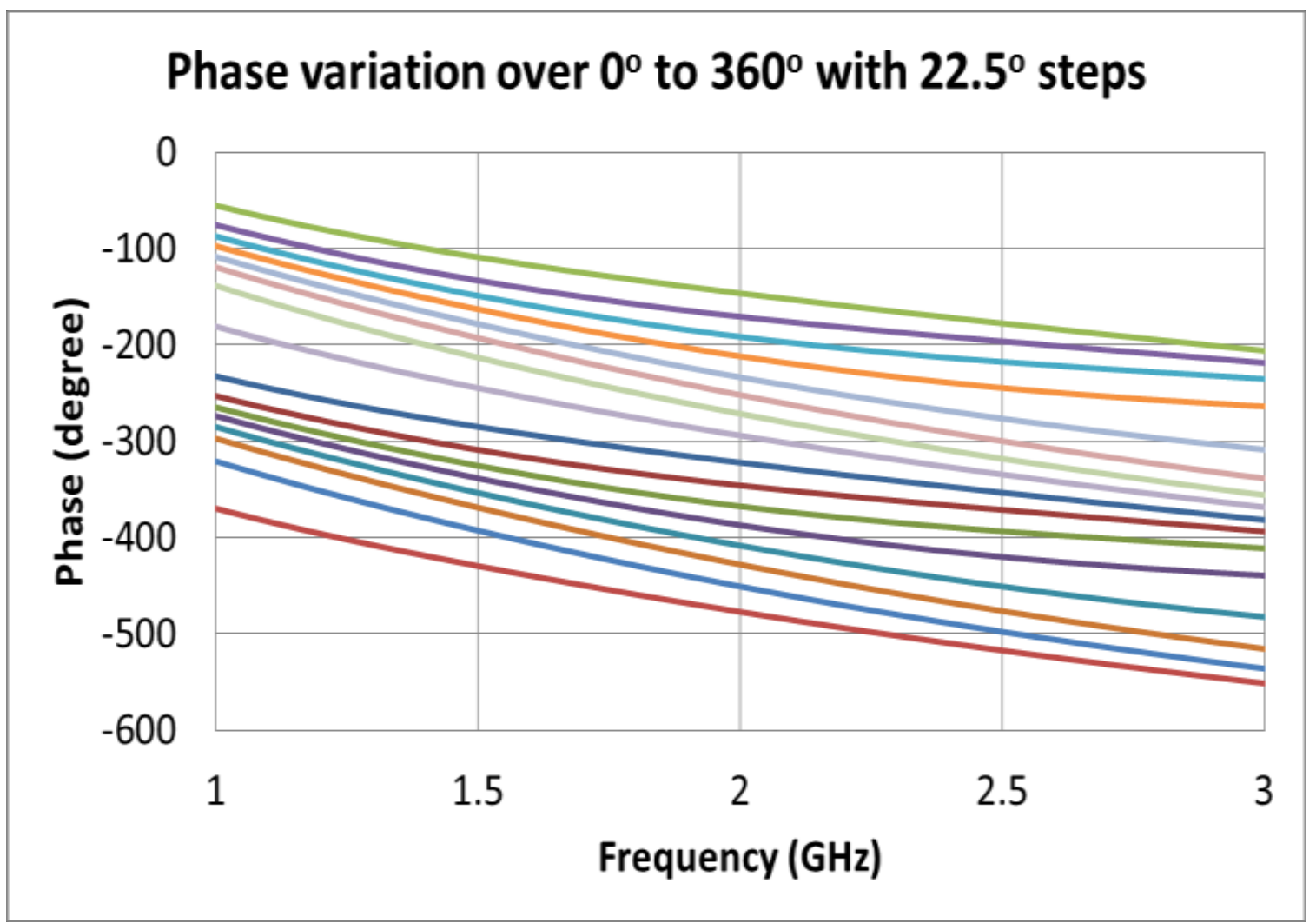

Figure 10: Frequency characteristics of phase variation in the entire $360^{\circ}$ range 


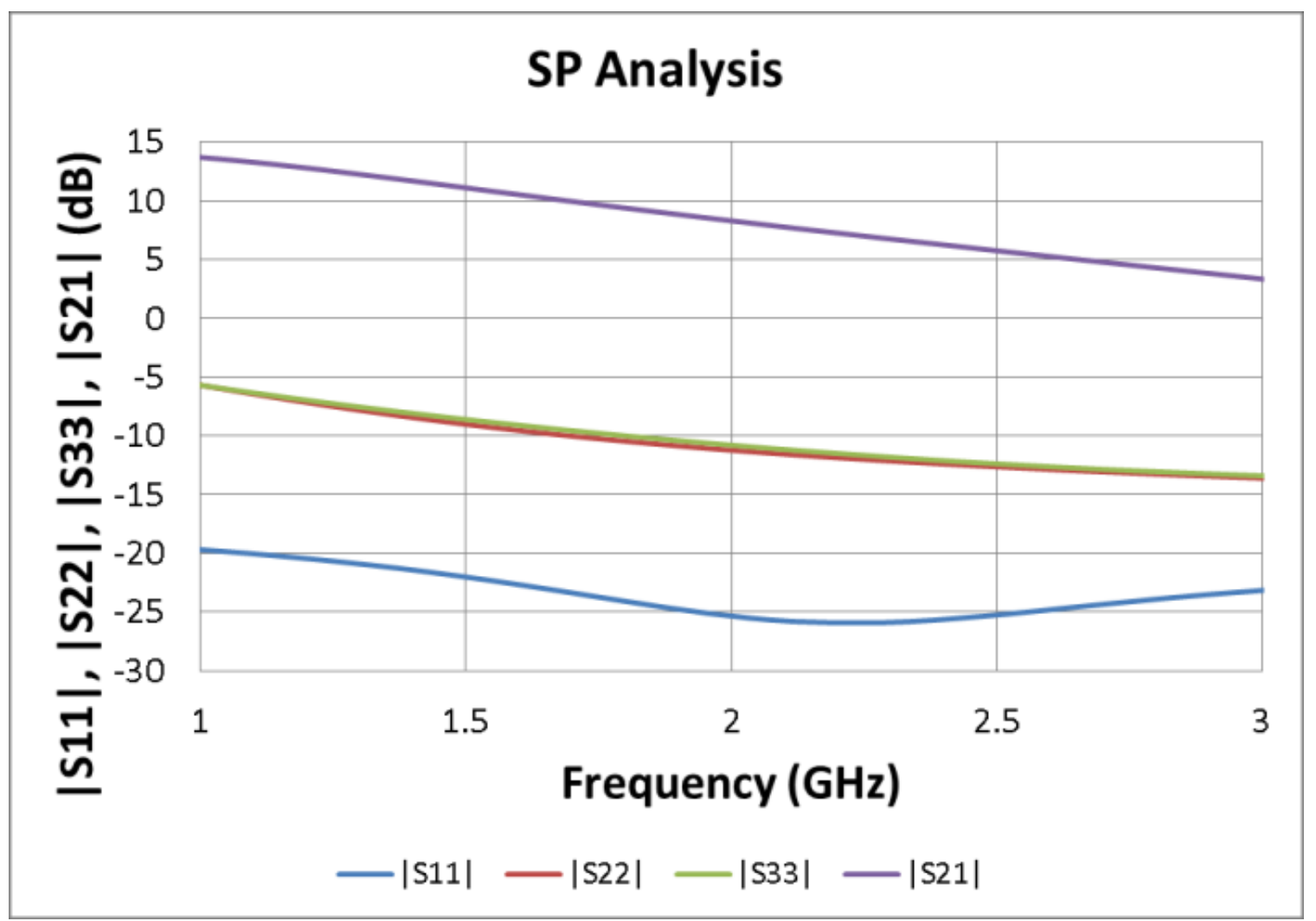

Figure 11: S-parameter analyses of conversion gain and return loss

The S-parameter simulation of the overall integrated phase shifter, with the input port (Port 1) in the passive hybrid and the two output ports (Port 2 and 3) in the two buffer circuits, is shown in Figure 11. We can see that the S-parameter analysis shows an input return loss (S11) better than $-25 \mathrm{~dB}$, an output return loss (S22) better than $-11 \mathrm{~dB}$ in the $2 \sim 3 \mathrm{GHz}$ and conversion gain (S21) of $7 \mathrm{~dB}$ achieved at $2.2 \mathrm{GHz}$. 


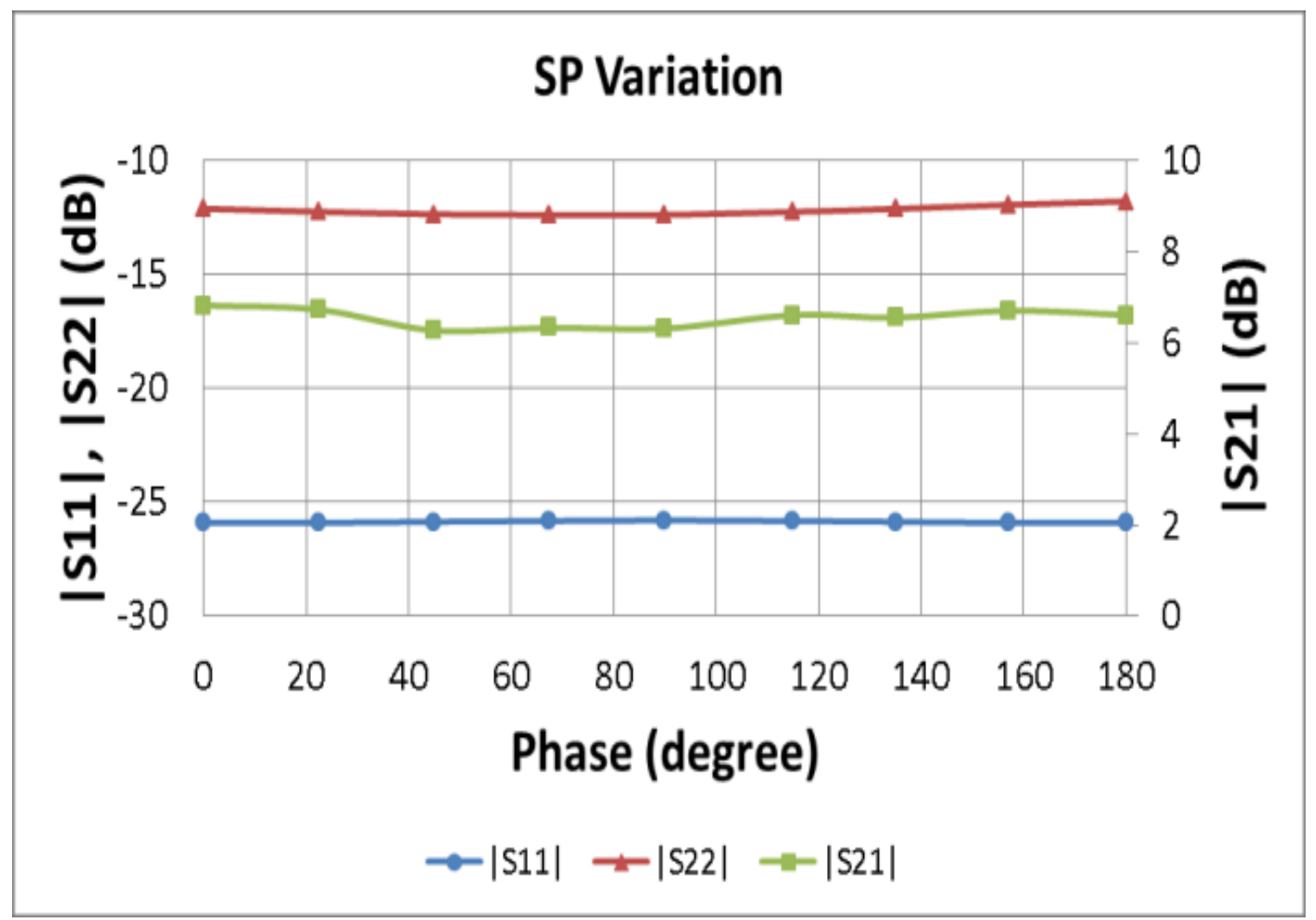

Figure 12: Stability of conversion gain and return loss over phase variation

Figure 12 shows very unique performances of this proposed CMOS integrated phase shifter where gain (S21) and input/output impedances $(\mathrm{S} 11, \mathrm{~S} 22)$ remain almost constant when phases are varied which is essential for simultaneous control of gain and phase variations without affecting each other. Large-signal performances are shown in Figure 13. 


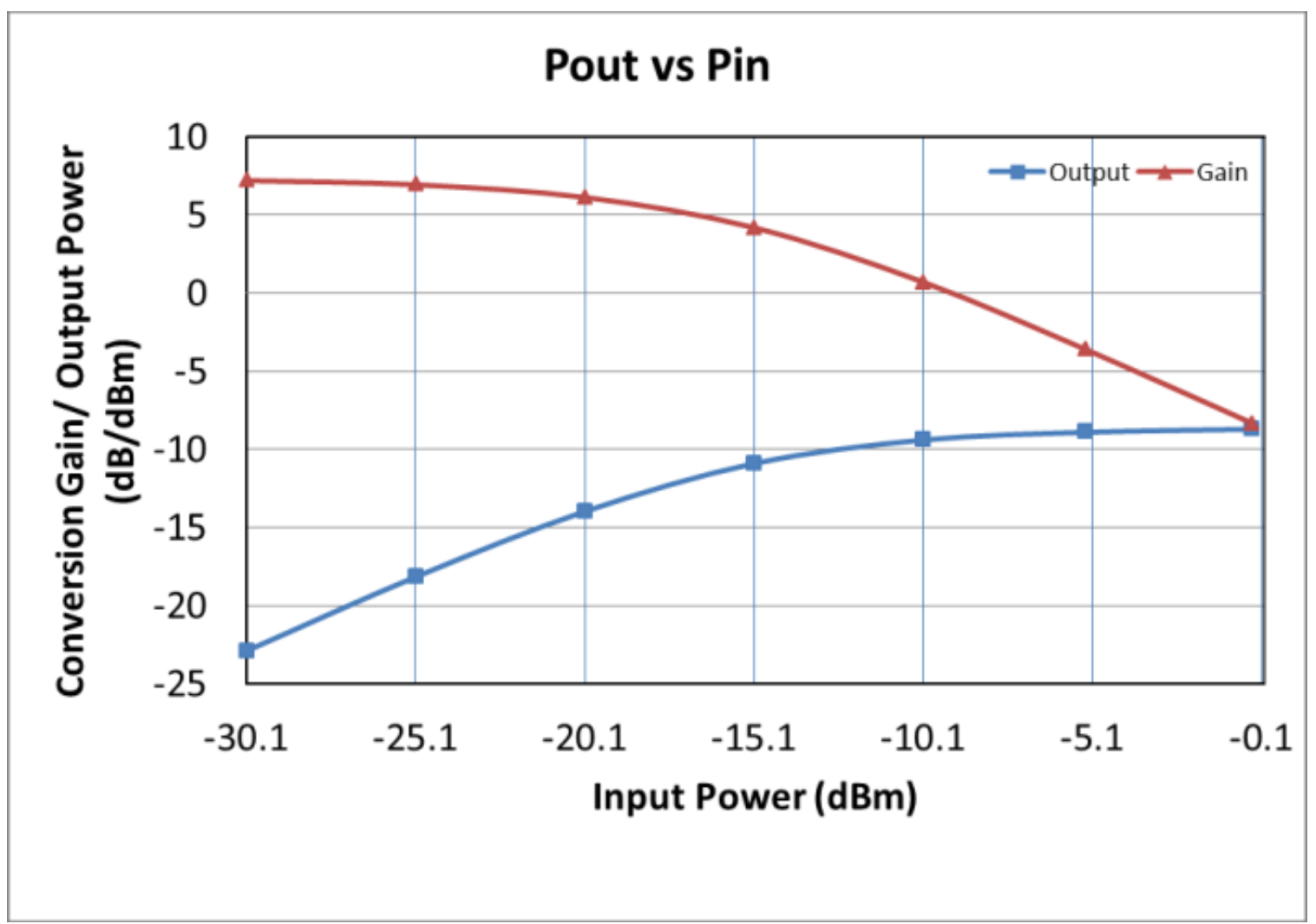

Figure 13: Large-signal performance showing compression of the integrated phase shifter

The 1-dB output compression power is found to be about $-15 \mathrm{dBm}$, which is sufficient for inclusion of this integrated phase shifter in the receiver circuitry and in the LO path of the transmitter circuitry. The layout of the integrated phase shifter with a size of $1.5 \mathrm{~mm} \mathrm{X} 0.75 \mathrm{~mm}$ including all bond pads is shown in Figure 14. Table 2 shows the performance comparison of our work with other phase shifters using various process technologies. 


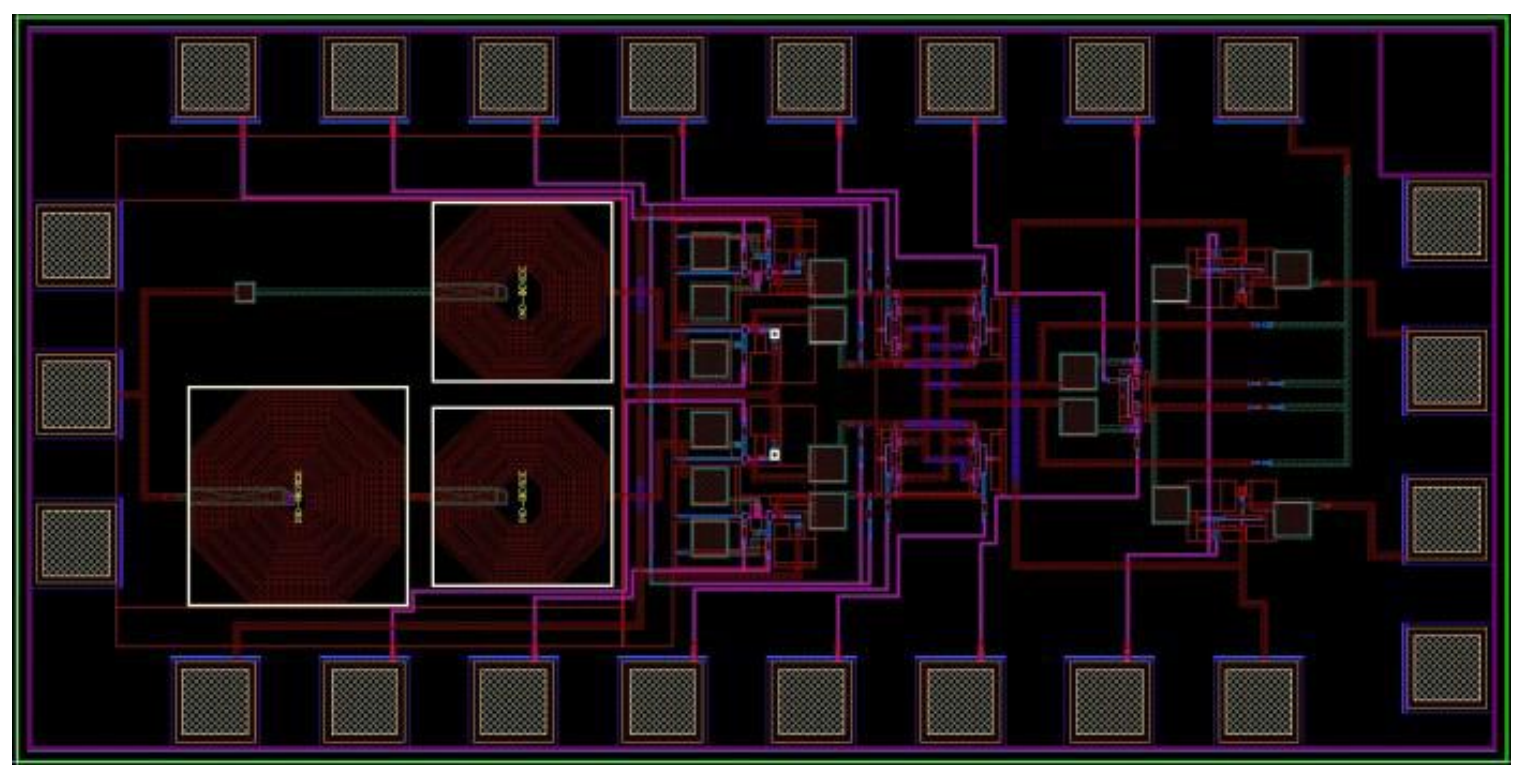

Figure 14: The layout of the phase shifter $(1.5 \mathrm{~mm} \mathrm{X} 0.75 \mathrm{~mm})$

Table 2: Performance Comparison

\begin{tabular}{|l|l|l|l|l|l|}
\hline Ref & {$[7]$} & {$[11]$} & {$[12]$} & {$[13]$} & This work \\
\hline $\begin{array}{l}\text { Frequency } \\
(\mathrm{GHz})\end{array}$ & $11-15$ & $15-26$ & $5.15-5.7$ & $4-6$ & $1-3$ \\
\hline $\begin{array}{l}\text { Conversion } \\
\text { Gain }(\mathrm{dB})\end{array}$ & -16.2 & -3.8 & -9 & -2.2 & $\begin{array}{l}+7.0 \\
\text { (variable) }\end{array}$ \\
\hline $\begin{array}{l}\text { Phase } \\
\text { Range }\left({ }^{\circ}\right)\end{array}$ & 360 & 360 & 360 & 90 & 360 \\
\hline Area $\left(\mathrm{mm}^{2}\right)$ & 4.34 & 0.15 & 0.9 & 0.5 & $\begin{array}{l}1.125 \text { (with } \\
\text { pads) }\end{array}$ \\
\hline $\begin{array}{l}\text { Power } \\
(\mathrm{mW})\end{array}$ & 0 & 11.7 & $\sim 0$ & $\sim 0$ & 17.48 \\
\hline $\begin{array}{l}\text { Process } \\
\text { Technology }\end{array}$ & $0.18 \mathrm{um}$ & $\begin{array}{l}.013 \mathrm{um} \\
\text { CMOS }\end{array}$ & $\begin{array}{l}0.6 \mathrm{~m} \text { GaAs } \\
\text { MESFET }\end{array}$ & $\begin{array}{l}0.6 \mathrm{~m} \text { GaAs } \\
\text { MESFET }\end{array}$ & $\begin{array}{l}0.18 \mathrm{um} \\
\mathrm{CMOS}\end{array}$ \\
\hline
\end{tabular}




\section{SUMMARY, CONTRIBUTIONS AND FUTURE WORK}

\subsection{Summary}

A vector modulator based CMOS phase shifter has been designed using 0.18um CMOS process. This phase shifter is integrated with an on-chip passive hybrid and active balun and is capable of achieving both gain/phase controls. This has potential application in integrating into a conformal phased array beamformer for recovering the degraded radiation pattern due to conformal shaping through simultaneous precise control of gain and phase. The results show stateof-the-art performances including conversion gain of $7 \mathrm{~dB}$ with variable feature for the first time to the best of author's knowledge. The simulation results, especially the plot of SP variation over the phase shift range over $360^{\circ}$ show that the gain (S21) and input and output impedances (S11, S22) remain fairly constant when there is a variation in phase which is essential for simultaneous control of gain and phase variations without affecting each other, within the range of 2-4GHz. The circuit can produce a continuous phase rotation of $360^{\circ}$ with steps as low as $11.25^{\circ}$ with total power consumption as low as $17 \mathrm{~mW}$.

\subsection{Contributions}

I proposed an integrated phase shifter architecture which is capable of controlling both phase and gain of the output signal. These two parameters are necessary to precisely restore the degraded radiation pattern of the conformal antenna array when it is being placed on a curved surface. The proposed architecture can keep constant phase when gain varies and keep constant 
gain when phase varies. This is essential so that both parameters can be used to correct the radiation pattern without being dependent on each other. In order to minimize power consumption, this integrated phase shifter is designed with co-design methodology where complex input/output impedances of each individual block is considered instead of standard $50 \Omega$ termination.

\subsection{Suggestions for Future Work}

The next steps of this project would be to characterize the fabricated chip and do the measurements. Once these are done, then an array of phase shifters can be realized. As part of our future endeavors, we intend to design the next step of phase shifters which can be used in closed loop circuits, receive a feedback from the output of the antenna and decide how much phase changed RF signal should be fed to the antenna as input signal in order to get the desired direction of the radiating beam of the antenna and also work on enhancing the output signal amplitude. 


\section{PUBLICATIONS RESULTING FROM THIS WORK}

[1] Alarka Sanyal, Alfonso Mendoza-Radal, Bilal Ijaz and Debasis Dawn, "CMOS Phase Shifter for Conformal Phased Array Beamformer Applications," presented at WiSEE 2013 IEEE International Conference.

[2] Bilal Ijaz, Alarka Sanyal, Alfonso Mendoza-Radal, Sayan Roy, Irfan Ullah, Michael T. Reich, Debasis Dawn and Benjamin D. Braaten, "Gain Limits of Phase Compensated Conformal Antenna Arrays on Non-Conducting Spherical Surfaces using the Projection Method,” presented at WiSEE 2013 IEEE International Conference.

[3] Alarka Sanyal, Alfonso Mendoza-Radal and Debasis Dawn, "CMOS Integrated Beamformer with Conformal Phased Array Antenna for Wireless Communications," poster presentation at IMAPS/NDSU Microelectronics Technology Summit, 2013. 


\section{REFERENCES}

[1] Koul, Shiban K., and Bharathi Bhat. Microwave and millimeter wave phase shifters: dielectric and ferrite phase shifters. Artech House, 1991.

[2] B. D. Braaten, M. A. Aziz, S.Roy, S. Nariyal, I. Irfanullah, N. F. Chamberlain, M. T. Reich and D. E. Anagnostou, "A Self-Adapting Flexible (SELFLEX) Antenna Array for Changing Conformal Surface Applications," IEEE Transactions on Antennas and Propagation, vol. 61, no. 2, Feb., 2013, pp. 655 - 665.

[3] Vadivelu, Praveen Babu, et al. "Integrated CMOS mm-wave phase shifters for single chip portable radar." Microwave Symposium Digest, 2009. MTT'09. IEEE MTT-S International. IEEE, 2009.

[4] "Various Types of Phase Shifter Circuit Services: An Understanding," microwavephaseshifter.wordpress.com/2013/03/13/various-types-of-phase shifter-circuitservices-an-understanding/

[5] Nagra, Amit S., and Robert A. York. "Distributed analog phase shifters with low insertion loss." Microwave Theory and Techniques, IEEE Transactions on 47.9 (1999): 1705-1711.

[6] Masaki, Ichiro, et al. "New architecture paradigms for analog VLSI chips."Vision Chips, Implementing Vision Algorithms Using Analog VLSI Circuits(1995): 353-375.

[7] Kang, Dong-Woo, et al. "Ku-band MMIC phase shifter using a parallel resonator with 0.18-/spl mu/m CMOS technology." Microwave Theory and Techniques, IEEE Transactions on 54.1 (2006): 294-301. 
[8] Yu, Yikun, et al. "A 60GHz digitally controlled phase shifter in CMOS." Solid-State Circuits Conference, 2008. ESSCIRC 2008. 34th European. IEEE, 2008.

[9] Malczewski, A., et al. "X-band RF MEMS phase shifters for phased array applications." Microwave and Guided Wave Letters, IEEE 9.12 (1999): 517-519.

[10] Cadence spectre/virtuoso Simulator by Cadence Design Systems, Inc., 2655 Seely Avenue, San Jose, CA 95134.

[11] Koh, Kwang-Jin; \& Rebeiz, Gabriel M.(2007). 0.13-mu m CMOS phase shifters for X-, Ku-, and K-band phased arrays.IEEE Journal of Solid-State Circuits, 42(11), 2535 - 2546.

[12] Ellinger, Frank, Rolf Vogt, and Werner Bachtold. "Ultracompact reflective-type phase shifter MMIC at C-band with 360 phase-control range for smart antenna combining." Solid-State Circuits, IEEE Journal of 37.4 (2002): 481-486.

[13] Ellinger, Frank, Rolf Vogt, and W. Bachtold. "Ultra compact, low loss, varactor tuned phase shifter MMIC at C-band." Microwave and Wireless Components Letters, IEEE 11.3 (2001): 104-105.

[14] K. Miyaguchi, et al., "An Ultra-Broad-Band Reflection-Type Phase-Shifter MMIC With Series and Parallel LC Circuits," IEEE Trans. Microw. Theory Tech., vol. 49, no. 12, pp. 2446-2452, Dec. 2001.

[15] Lu, Liang-Hung, and Yu-Te Liao. "A 4-GHz phase shifter MMIC in $0.18-\mu \mathrm{m}$ CMOS." Microwave and Wireless Components Letters, IEEE 15.10 (2005): 694-696.

[16] M.-D. Tsai and H. Wang, "A 0.3-25-GHz ultra-wideband mixer using commercial 0.18_m CMOS technology,” IEEE Microw. Wireless Compon. Lett., vol. 14, no. 11, pp. 522524, Nov. 2004.

[17] J. O. Plouchart, J. Kim, N. Zamdmer, L.-H. Lu, M. Sherony, Y. Tan, R. Groves, R. 
Trizcinski, M. Talbi, A. Ray, and L. Wagner, “A 4-91-GHz traveling-wave amplifier in a standard 0.12-_m SOI CMOS microprocessor technology,” IEEE J. Solid-State Circuits, vol. 39, no. 11, pp. 1455-1461, Sep. 2004.

[18] A. S. Nagra and R. A. York, "Distributed analog phase shifters with low insertion loss," IEEE Trans. Microw. Theory Tech., vol. 47, no. 9, pp. 1705-1711, Sep. 1999.

[19] S. Lee, J.-H. Park, H.-T. Kim, J.-M. Kim, Y.-K. Kim, and Y. Kwon, "Low-loss analog and digital reflection-type MEMS phase shifters with $1: 3$ bandwidth,” IEEE Trans. Microw. Theory Tech., vol. 52, no. 1, pp. 211-219, Jan. 2004.

[20] D. C. Boire, G. S. Onge, C. Barratt, G. B. Norris, and A. Moysenko, "4 : 1 bandwidth digital five bit MMIC phase shifters," in Microwave Millimeter-Wave Monolithic Circuit Symp. Dig., Jun. 1989, pp. 69-73.

[21] H. Hayashi, T. Nakagawa, and K. Araki, “A miniaturized MMIC analog phase shifter using two quarter-wave-length transmission lines," IEEE Trans. Microw. Theory Tech., vol. 50, no. 1, pp. 150-154, Jan. 2001.

[22] H. Zarei and D. J. Allstot, "A low-loss phase shifter in $180 \mathrm{~nm}$ CMOS for multiple-antenna receivers," in IEEE Int. Solid-State Circuits Conf. Tech. Dig., vol. 1, Feb. 2004, pp. 392393.

[23] H. D. Lee and D. W. Kang, "A Ku-band MOSFET phase shifter MMIC,” in IEEE MTT-S Int. Microwave Symp. Dig., Jun. 2004, pp. 191-194.

[24] M. Kumar, R. J. Menna, and H. Huang, "Broad-band active phase shifters using dual gate MESFET," IEEE Trans. Microw. Theory Tech., vol. MTT-29, no. 10, pp. 1098-1101, Oct. 1981.

[25] Y. Gazit and H. C. Johnson, "A continuously-variable Ku-band phase/amplitude control 
module," in IEEE MTT-S Int. Microwave Symp. Dig., 1981, pp. 436-438.

[26] J. Grajal, J. Gismero, M. Mahfoudi, and F. A. Petz, “A 1.4-2.7-GHz analog MMIC vector modulator for a crossbar beamforming network," IEEE Trans. Microw. Theory Tech., vol. 45, no. 10, pp. 1705-1714, Oct. 1997.

[27] J. R. Selin, "Continuously variable L-band monolithic GaAs phase shifter," Microwave J., vol. 30, pp. 211-218, Sep. 1987.

[28] D. K. Paul and P. Gardner, "Microwave quadrature active phase shifter using MESFETs," Microwave Opt. Technol. Lett., vol. 15, pp. 359-360, Aug. 1997.

[29] S. J. Kim and N. H. Myung, "A new active phase shifter using a vector sum method," IEEE Microw. Guided Wave Lett., vol. 10, no. 6, pp. 233-235, Jun. 2000.

[30] P.-Y. Chen, T.-W. Huang, H. Wang, Y.-C. Wang, C.-H. Chen, and P.-C. Chao, "K-band HBT and HEMT monolithic active phase shifters using vector sum method," IEEE Trans. Microw. Theory Tech., vol. 52, no. 5, pp. 1414-1424, May 2004.

[31] Wu, Pei-Si, et al. "New miniature 15-20-GHz continuous-phase/amplitude control MMICs using $0.18-\mu \mathrm{m}$ CMOS technology." Microwave Theory and Techniques, IEEE Transactions on 54.1 (2006): 10-19.

[32] Sarkas, I., et al. "W-band 65-nm CMOS and SiGe BiCMOS transmitter and receiver with lumped IQ phase shifters." Radio Frequency Integrated Circuits Symposium, 2009. RFIC 2009. IEEE. IEEE, 2009.

[33] R. G. Stewart and M. N. Giuliano, "X band integrated diode phase shifter," in G-MTT Int. Microwave Symp. Dig.,May 1968, pp. 147-154.

[34] R. W. Burns and R. L. Holden, "Acompact S-band diode phase shifter," in G-MTT Int. Microwave Symp. Dig., Jun. 1973, pp. 298-300. 
[35] R. P. Coats, "An octave-band switched-line microstrip 3-b diode phase shifter," IEEE Trans. Microw. Theory Tech., vol. MTT-21, no. 7, pp. 444-449, Jul. 1973.

[36] R. N. Hardin, E. J. Downey, and J. Munushian, "Electronically variable phase shifter utilizing variable capacitance diodes,” Proc. Inst. Radio Eng., vol. 48, pp. 944-945, May 1960.

[37] J. F. White, "Diode phase shifters for array antennas," IEEE Trans. Microw. Theory Tech., vol. MTT-22, no. 6, pp. 658-674, Jun. 1974.

[38] J. P. Srarski, "Optimization of the matching network for a hybrid coupler phase shifter," IEEE Trans. Microw. Theory Tech., vol. MTT-25, no. 8, pp. 662-666, Aug. 1977.

[39] J. F. White, "High power, p-i-n diode controlled, microwave transmission phase shifters," IEEE Trans. Microw. Theory Tech., vol. MTT-13, no. 2, pp. 233-242, Mar. 1965.

[40] F. L. Opp and W. F. Hoffman, "Design of digital loaded-line phase shift networks for microwave thin-film applications," IEEE J. Solid-State Circuits, vol. SSC-3, no. 2, pp. 124-130, Jun. 1968.

[41] M. E. Davis, "Integrated diode phase-shifter elements for an X-band phased-array antenna," IEEE Trans. Microw. Theory Tech., vol. MTT-23, no. 12, pp. 1080-1084, Dec. 1975.

[42] F. Ellinger, H. Jackel, and W. Bachtold, "Varactor-loaded transmission- line phase shifter at C-band using lumped elements," IEEE Trans. Microw. Theory Tech., vol. 51, no. 4, pp. 1135-1140, Apr. 2003.

[43] T. M. Hancock and G. M. Rebeiz, "A 12-GHz SiGe phase shifter with integrated LNA," IEEE Trans. Microw. Theory Tech., vol. 53, no. 3, pp. 977-983, Mar. 2005.

[44] C. Lu, A.-V. H. Pham, and D. Livezey, "Development of multiband phase shifters in 180- 
nm RF CMOS technology with active loss compensation," IEEE Trans. Microw. Theory Tech., vol. 54, no. 1, pp. 40-45, Jan. 2006.

[45] F. Ali and A. Podell, "A wideband GaAs monolithic spiral quadrature hybrid and its circuit applications," IEEE J. Solid-State Circuits, vol. 26, no. 10, pp. 1394-1398, Oct. 1991.

[46] Y. Ayasli, S. W. Miller, R.Mozzi, and L. K. Hanes, "Wide-band monolithic phase shifter," IEEE Trans. Microw. Theory Tech., vol. MTT-32, no. 12, pp. 1710-1714, Dec. 1984.

[47] C. F. Campbell and S. A. Brown, "A compact 5-bit phase-shifter MMIC for K-band satellite communication systems," IEEE Trans. Microw. Theory Tech., vol. 48, no. 12, pp. 2652-2656, Dec. 2000.

[48] D.-W. Kang, H.-D. Lee, C.-H. Kim, and S. Hong, "Ku-band MMIC phase shifter using a parallel resonator with 0.18__m CMOS tehcnology,” IEEE Trans. Microw. Theory Tech., vol. 54, no. 1, pp. 294-301, Jan. 2006.

[49] M. I. Skolnik, Introduction to RADAR Systems, 3rd ed. New York: McGraw-Hill, 2001.

[50] N. Imai and H. Ichikawa, "One-chip endless phase shifter IC's for space diversity combiner," IEEE Trans. Circuits Syst. II, Analog Digit. Signal Process., vol. 43, no. 4, pp. 281-288, Apr. 1996.

[51] M. Chua and K. W. Martin, "1 GHz programmable analog phase shifter for adaptive antennas," in Proc. IEEE Custom Integrated Circuits Conf., 1998, pp. 71-74.

[52] A. Natarajan, A. Komijani, X. Guan, A. Babakhani, Y. Wang, and A. Hajimiri, “A 77 GHz phased-array transmitter with local LO-path phase-shifting in silicon,” in IEEE Int. SolidState Circuits Conf. Dig. Tech. Papers, 2006, pp. 639-648.

[53] X. Yang and J. Lin, "A digitally controlled constant envelop phaseshift modulator for lowpower broadband wireless applications," IEEE Trans. Microw. Theory Tech., vol. 54, no. 
1, pp. 96-105, Jan. 2006.

[54] J. Paramesh, K. Soumyanath, and D. J. Allstot, “A four-antenna receiver in 90-nm CMOS for beamforming and spatial diversity," IEEE J. Solid-State Circuits, vol. 40, no. 12, pp. 2515-2524, Dec. 2005.

[55] K.-J. Koh, M.-Y. Park, C.-S. Kim, and H.-K. Yu, "Subharmonically pumped CMOS frequency conversion (up and down) circuits for 2-GHz WCDMA direct-conversion transceiver,” IEEE J. Solid-State Circuits, vol. 39, no. 6, pp. 871-884, Jun. 2004. 